\title{
理想 $\operatorname{Im} \sigma_{*}^{2 k}$ 的群结构
}

$M O_{n-2 k}(B O(2 k+1))$ 是 $n-2 k$ 维 光滑闭流形上实 $(2 k+1)$ 维平面丛的末定 向上协边群, $M O$, 是末定向上协边群.

$$
\sigma_{n}^{2 k}: M O_{n-2 k}(B O(2 k+1)) \rightarrow M O_{n}
$$

是一个群同态, 它把 $M^{n-2 k}$ 上的 $2 k+1$ 维 平面丛映射到联系射影空间丛的全空间的上 协边类. $\operatorname{Im} \sigma_{*}^{2 k}=\sum_{n<2 k} \operatorname{Im} \sigma_{*}^{2 k}$ 是由 Stong 流 形 $R P\left(n_{1}, n_{2}, \cdots, n_{2 k+1}\right)$ 的上协边类生成的 $M O_{*}$ 中一个理想.

设

$$
\begin{aligned}
& x_{2}=[R P(2)] \text {, } \\
& x_{4}-[R P(4)] \text {, } \\
& x_{j}=[R P(j-3,0,0,0)], j-5,6 \text {, } \\
& x_{9}=[R P(2, \underbrace{0, \cdots, 0}_{7})] \text {, } \\
& x_{11}-[R P(4, \underbrace{0, \cdots, 0})] \text {, } \\
& x_{13}-[R P(3,2, \underbrace{0, \cdots, 0}_{7})] \text {, } \\
& x_{17}=[R P(7,2, \underbrace{0, \cdots, 0}_{7}) \text {, } \\
& x_{r}-\left[R P(\frac{r}{2}-4, \frac{r}{2}-4, \underbrace{0, \ldots, 0}_{r})\right] \text {, } \\
& r-8,10,12,14,16,18 \text {. } \\
& \text { 令 } \\
& B_{1}=\varnothing ; \\
& B_{2}=\{[R P(1,1,0,0,0)]\} \text {, } \\
& B_{3}=\{[R P(6)][R P(2, \underbrace{0, \cdots, 0}_{6})], \\
& {[R P(2,1,0, \ldots, 0)],} \\
& {[R P(1,1,1,1,0,0,0)] \text {, }} \\
& x_{2}^{3}+x_{s}^{2},[R P(4,1, \underbrace{0, \cdots, 0)}_{5}] \text {, } \\
& \left.x_{2}^{3} x_{3}+x_{2} x_{4} x_{3},[R P(\underbrace{1, \cdots, 1}, 0)]\right\} \text {. }
\end{aligned}
$$

$$
\begin{aligned}
& \text { B，是由下列上协边类构成的集合: } \\
& x_{8}, x_{10}, x_{2}^{3} x_{5}+x_{5} x_{4} x_{2}+x_{6} x_{5}+x_{9} x_{2} \text {, } \\
& x_{12}, x_{6}^{2}+x_{5}^{2} x_{2}+x_{4} x_{2}^{4} \text {, } \\
& x_{13}, x_{9} x_{2}^{2}+x_{6} x_{5} x_{2}+x_{5} x_{4} x_{2}^{2}+x_{5} x_{2}^{4}, \\
& x_{11} x_{2}+x_{9} x_{4}+x_{9} x_{2}^{2}+x_{5} x_{4} x_{2}^{2}+x_{5} x_{2}^{4} \text {, } \\
& x_{14}, x_{5}^{2} x_{4}, x_{9} x_{5}+x_{6} x_{4}^{2}+x_{6} x_{4} x_{2}^{2} \\
& +x_{1}^{2} x_{2}^{3}+x_{1} x_{2}^{5} \text {, } \\
& x_{6} x_{2}^{4}+x_{5}^{2} x_{2}^{2}+x_{2}^{7} \text {, } \\
& x_{6}^{2} x_{2}+x_{5}^{2} x_{2}^{2}+x_{4} x_{2}^{5} \text {, } \\
& x_{11} x_{9}+x_{11} x_{2}^{2}+x_{9} x_{6}+x_{5} x_{1}^{2} x_{2}+x_{5} x_{2}^{\prime} \text {, } \\
& x_{9} x_{6}+x_{9} x_{6} x_{2}+x_{6} x_{5} x_{2}^{2}+x_{5}^{3}+x_{5} x_{4} x_{2}^{3} \\
& +x_{3} x_{2}^{5} \\
& x_{11} x_{4}+x_{9} x_{6}+x_{6} x_{5} x_{4}+x_{6} x_{5} x_{2}^{2}+x_{5}^{3} \\
& +x_{5} x_{4} x_{2}^{3} \\
& x_{11} x_{4}+x_{11} x_{2}^{2}+x_{9} x_{4} x_{2}+x_{6} x_{5} x_{2}^{2} \\
& +x_{5} x_{4}^{2} x_{2}+x_{5} x_{4} x_{2}^{3}+x_{5} x_{2}^{5} \text {, } \\
& x_{11} x_{4}+x_{9} x_{4} x_{2}+x_{6} x_{5} x_{4}+x_{5}^{3} \text {, } \\
& x_{9} x_{6}+x_{9} x_{2}^{3}+x_{9}^{3}+x_{5} x_{2}^{3}, x_{9} x_{6}+x_{9} x_{2}^{3} \text {; } \\
& x_{16}, x_{9} x_{5} x_{2}, x_{6}^{2} x_{4}, x_{0}^{2} x_{2}^{2}, x_{5}^{2} x_{4} x_{2}, x_{4}^{4} \text {, } \\
& x_{1}^{2} x_{2}^{4}, x_{2}^{2}, x_{11} x_{5}+x_{6} x_{4}^{2} x_{2}+x_{6} x_{4} x_{2}^{3} \\
& +x_{5}^{2} x_{2}^{4}+x_{4} x_{2}^{n} \text {, } \\
& x_{6} x_{3}^{2}+x_{3}^{2} x_{2}^{1}, x_{6} x_{4}^{2} x_{2}+x_{6} x_{4} x_{2}^{2}+x_{4} x_{2}^{6} \text {, } \\
& x_{6} x_{2}^{2}+x_{6} x_{2}^{\mathrm{p}}, x_{5}^{2} x_{2}^{3}+x_{4} x_{2}^{2}, x_{6} x_{4}+x_{1}^{4} x_{2}^{3},
\end{aligned}
$$

不包含 $x_{5} x_{4}^{\prime}, x_{6} x_{4}^{3}$ 和 $x_{4}^{3} x_{2}^{3}$ 的 17,18 维所有 上协边类.

$$
\text { 令 } n_{1}-2, n_{2}-7, n_{3}-13, n_{4}-19 \text {. }
$$

定理 设 $\gamma_{n} \in M O_{\text {.. }} \gamma_{n}$ 属于 $\operatorname{Im} \sigma_{*}^{2 k}$ $(k-1,2,3,4)$ 的充要条件是 $n \geqslant n_{k}$ 或 者 $n<n_{k}$, 但 $\gamma_{n}$ 可分解为 $B_{k}$ 中元作为因 子的单项式的线性组合. 即

$$
\operatorname{Im} \sigma_{*}^{2 k}-\left\{\sum a_{n, m} \alpha_{m} \beta_{m} \mid a_{n, m} \in Z_{2}, \quad n+m\right.
$$
$\geqslant n_{k}, \alpha_{n}, \beta_{m} \in M O_{*}$ 或者 $n+m<n_{k}$,

$$
\text { 通报 }
$$


$\left.a_{n} \in B_{k}, \beta_{m} \in M O_{m}\right\}, k-1,2,3,4$.

\section{考文 韵}

[1] Stong, R. E., Trans. Amer. Math. Soc., 178 (1973), 431-447.
[2]王彦英,数学学报, 31 (1988), 2: 158-163.

蒋国瑞

(河北师范学院数学研究所, 石家庄 050091)

王彦英

（河北师范大学数学系, 石家庄 050016）

\section{关于 Fisher 方程的孤波解}

文献[ 1 ] 给出了 Fisher 方程

$$
\frac{\partial u}{\partial t}-\frac{\partial^{2} u}{\partial x^{2}}-u\left(1-u^{a}\right)
$$

的显式精确孤波解. 本文旨在利用一个新的 变换给出原方程的另一组解. 做三角变换

方程 (1) 变为

$$
u-\sin ^{\frac{1}{a}} w \text {, }
$$

$$
\begin{aligned}
& \frac{\partial w}{\partial t}-\frac{\partial^{2} w}{\partial x^{2}}-\left[2 \operatorname{ctg} 2 w+2\left(\frac{2}{\alpha}-1\right)\right. \\
& \times \operatorname{ctg} w]\left(\frac{\partial w}{\partial x}\right)^{2}-\frac{\alpha}{8} \sin 2 w\left(1+\sin ^{2} w\right) .
\end{aligned}
$$

再设方程 (1) 有行波解

$$
w(x, t)-w(x-c t)=w(\xi), c>0 .
$$

方程 (3) 变为

$$
\begin{gathered}
-c \frac{d w}{d \xi}-\frac{d^{2} w}{d \xi^{2}}-\left[2 \operatorname{ctg} 2 w+2\left(\frac{2}{\alpha}-1\right)\right. \\
\times \operatorname{ctg} w]\left(\frac{d w}{d \xi}\right)^{2}-\frac{\alpha}{8} \sin 2 w \\
\times\left(1+\sin ^{2} w\right) .
\end{gathered}
$$

进一步假设

$$
\frac{d w}{d \xi}-a \sin 2 w,
$$

上式 $a$ 为待定参数. 我们有

$$
d^{2} w / d \xi^{2}=2 a^{2} \sin 2 w \cos 2 w \text {. }
$$

从而方程 (3) 变为

$$
\begin{aligned}
& -c a \sin 2 w-2 a^{2} \sin 2 w \cos 2 w \\
& -\left[2 \operatorname{ctg} 2 w+2\left(\frac{2}{\alpha}-1\right) \operatorname{ctg} w\right] \\
& \times a^{2} \sin 2 w-\frac{\alpha}{8} \sin 2 w \\
& \times\left(1+\sin ^{2} w\right) .
\end{aligned}
$$

相应系数的比较给出 $a=\frac{-\alpha}{4 \sqrt{\alpha+4}}$,

$$
c-\frac{16+\alpha}{4 \sqrt{\alpha+4}} .
$$

方程 (5) 的积分给出 $\operatorname{tg} w-\mathrm{e}^{2 a \xi+b}$. $b$ 为积 分常数. 回到变换 (2), 即给出原方程的孤 波解

$u(x, t)=$

$\left\{\frac{\operatorname{tash}\left[\frac{-\alpha}{2 \sqrt{\alpha+4}}\left(x-\frac{16+\alpha}{4 \sqrt{\alpha+4}} t\right)+b\right]+1}{2}\right\}^{2 / a}$.

上述解对于讨论向非稳态过渡的一类物理问 题是有启发意义的。

\section{考文 献}

[1]王心宜,科学通报, 33 (1988), 9: 657.

王心宜

（苏州大学物理系,苏州 215006) 